\title{
Cytochrome $\mathbf{C}$ inhibits tumor growth and predicts favorable prognosis in clear cell renal cell carcinoma
}

\author{
ZHIGUO LIU ${ }^{1}$, XIANCHENG ZHAO ${ }^{2}$, LIANG ZHANG ${ }^{3}$ and BING PEI ${ }^{4}$ \\ Departments of ${ }^{1}$ Hospital Pharmacy, ${ }^{2}$ Urinary Surgery, ${ }^{3}$ Nephrology and ${ }^{4}$ Clinical Laboratory, \\ Suqian First Hospital, Suqian Branch Jiangsu Province Hospital, Suqian, Jiangsu 223800, P.R. China
}

Received February 17, 2019; Accepted August 20, 2019

DOI: $10.3892 / \mathrm{ol} .2019 .10989$

\begin{abstract}
Cytochrome C (Cyto C), a multifunctional enzyme, has been demonstrated to be associated with cell apoptosis and respiration. Accumulating evidence has revealed that serum Cyto $\mathrm{C}$ is an effective indicator in evaluating the effect of chemotherapy. However, to the best of our knowledge, the clinical significance of Cyto $\mathrm{C}$ and its role in cell growth and apoptosis in clear cell renal cell carcinoma (CCRCC) remain unknown. In the present study, Cyto $\mathrm{C}$ expression was detected in 150 CCRCC and 30 normal tissues samples via immunohistochemistry. The results demonstrated that Cyto $\mathrm{C}$ protein expression levels in CCRCC tissues were downregulated compared with those in corresponding normal tissues. In addition, it was revealed that $\mathrm{Cy}$ to $\mathrm{C}$ expression was negatively associated with TNM stage. Further analyses revealed that patients with CCRCC and low Cyto $\mathrm{C}$ expression levels had a shorter survival time than those with high Cyto $\mathrm{C}$ expression. Multivariate analyses indicated that high Cyto $\mathrm{C}$ expression levels were an independent prognostic factor for survival. Functionally, overexpression of Cyto $\mathrm{C}$ effectively suppressed the growth of CCRCC cells and induced cell apoptosis, and knockdown of Cyto $\mathrm{C}$ reversed these effects. Finally, overexpression of Cyto $\mathrm{C}$ inhibited the tumor growth of CCRCC cells in vivo. Overall, the data of the present study indicated that Cyto $\mathrm{C}$ may be a novel prognostic biomarker and acted as a regulator of tumor growth in CCRCC.
\end{abstract}

\section{Introduction}

Clear cell renal cell carcinoma (CCRCC) represents the most common subtype of renal cancer, which is frequently observed to exhibit alterations in the von Hippel-Lindau gene (1). Although nephrectomy, radiotherapy and immunotherapy

Correspondence to: Dr Xiancheng Zhao, Department of Urinary Surgery, Suqian First Hospital, Suqian Branch Jiangsu Province Hospital, 120 Suzhilu, Suqian, Jiangsu 223800, P.R. China

E-mail: xczhaosq@163.com

Key words: apoptosis, clear renal cell carcinoma, cytochrome C, proliferation have been applied in the treatment of CCRCC, the prognosis of advanced CCRCC remains poor, due to a lack of early symptoms, signs and laboratory abnormalities (2). Therefore, it is worth focusing on novel biomarkers that are involved in the carcinogenic process of CCRCC development.

Cytochrome $\mathrm{C}$ (Cyto $\mathrm{C}$ ), located on the inner surface of mitochondria, is a heme-containing metalloprotein and multifunctional enzyme, that is involved in cell apoptosis (3). As a mitochondrial biomarker, Cyto $\mathrm{C}$ is released from mitochondria into the extracellular space and the bloodstream via permeabilization of injured mitochondria within $1 \mathrm{~h}$ of apoptosis induction (4). Therefore, Cyto $\mathrm{C}$ is considered to be a critical mediator and biomarker in mitochondria-mediated apoptosis (5). Furthermore, the translocation of Cyto C is a rapid and apoptosis-specific process, which occurs not only under normal physiological conditions but also exists in patients with cancer (6). Previous studies have demonstrated that serum Cyto $\mathrm{C}$ is an indicator of outcome during therapy of various types of cancer, including leukemia and lung cancer $(6,7)$, indicating that $\mathrm{Cy}$ to $\mathrm{C}$ may be involved in tumor initiation and progression. Nevertheless, the clinical relevance and function of Cyto $\mathrm{C}$ in $\mathrm{CCRCC}$ remain to be elucidated.

\section{Materials and methods}

Cell culture. The CCRCC cell line 786-O was purchased from the American Type Culture Collection (cat. no. CRL-1932), and cultured in DMEM (HyClone; GE Healthcare Life Sciences) supplemented with $10 \%$ FBS. The cells were incubated in a humidified incubator at $37^{\circ} \mathrm{C}$ with $5 \% \mathrm{CO}_{2}$.

Clinical samples. In the present study, 10 pairs of tumor and corresponding normal tissues were obtained from patients with CCRCC (4 women and 6 men; mean age, 51.2 \pm 3.1 years), who underwent surgical resection at the Suqian First Hospital (Suqian, China) between January 2016 and December 2017. Normal tissues were isolated $5 \mathrm{~cm}$ away from the corresponding tumor margin. Tissue microarrays, including 150 CCRCC and 30 corresponding normal tissues, were obtained from Shanghai BioChip Co Ltd. Clinical information for the specimens included in the tissue microarray is presented in Table SI. The Tumor-Node-Metastasis staging (TNM; American Joint Committee on Cancer, 2017) and grading 
Fuhrman grading systems were used for the classification of patients with CCRCC (8).

Gene Expression Omnibus (GEO) database. The mRNA levels of Cyto $\mathrm{C}$ in CCRCC were publicly available from the National Center for Biotechnology Information GEO database (accession no. GDS505) (9). The mRNA levels of Cyto C were compared using a paired Student's t-test.

IHC staining and scoring. Slides of human tissue microarrays were deparaffinized at $60^{\circ} \mathrm{C}$ by a series of xylene and decreasing gradient of ethanol $(100,95,85$ and $75 \%)$ and heated in $10 \mathrm{mM}$ citrate buffer, $\mathrm{pH} 6.0$, for $15 \mathrm{~min}$ in a microwave oven. Endogenous peroxidase activity was blocked with $0.3 \%$ hydrogen peroxide and $0.1 \%$ saponin dissolved in TBS for $30 \mathrm{~min}$ at $37^{\circ} \mathrm{C}$. Sections were incubated with mouse polyclonal anti-Cyto $\mathrm{C}$ (1:100; cat. no. ab133504; Abcam) at $4^{\circ} \mathrm{C}$, followed by incubation with avidin-biotin-peroxidase at $37^{\circ} \mathrm{C}(1: 1$; cat. no. COD-Nr5007; Dako; Agilent Technologies, Inc.). Sections were stained with 3-diaminobenzidine and scored for the level of Cyto $\mathrm{C}$ expression using the following system: $0,<1 \%$ cells with positive staining; $1,1-25 \%$ positive cells; $2,26-50 \%$ positive cells; $3,51-75 \%$ positive cells; and $4,76-100 \%$ positive cells. The staining intensity of Cyto C was scored as follows: 0 , negative; 1 weak; 2 , moderate; and 3, high intensity (Fig. S1). The final score of Cyto C expression was defined as the staining intensity score multiplied by the score for the percentage of positive cells. The cut-off value of the IHC score was determined using X-tile statistical software version 3.6.1 (10). All slides were evaluated using a light microscope independently by two pathologists who were blinded to the clinical outcome of the patients. The present study was performed according to the principles of the Declaration of Helsinki and approved by the Ethics Committee of Suqian First Hospital.

Validation analysis using the TCGA database. The clinicopathological information and expression data of the patients with CCRCC from The Cancer Genome Atlas (TCGA)-database (http://www.tcga.org/) were downloaded from The Human Protein Atlas database (https://www.proteinatlas.org).

Lentiviral infection and small interfering (si)RNA tranfection procedures. For overexpression of Cyto $\mathrm{C}$ in CCRCC cells, the CytoCcDNA wasclonedinto apLOV-CMV-eGFP-EF1a-PuroR lentiviral vector (GeneChem, Inc.). Lentiviral particles were packaged using Helper 1.0. CCRCC cells were infected with concentrated Cyto C-flag lentivirus (MOI=2), and then selected and enriched with $4 \mu \mathrm{g} / \mathrm{ml}$ puromycin for a week, and $2 \mu \mathrm{g} / \mathrm{ml}$ puromycin was sustained for continuous selection pressure (termed the OECyto $\mathrm{C}$ line). The mock group was infected with empty lentivirus.

For the knockdown experiments, 786-O cells were transfected with siRNAs targeting Cyto C (15 nM; cat. no. sc-29292; Santa Cruz Biotechnology, Inc.) and control siRNAs (siNC; cat. no. sc-37007; Santa Cruz Biotechnology, Inc.) using Lipofectamine ${ }^{\circledR} 3000$ (Invitrogen; Thermo Fisher Scientific, Inc.). After $6 \mathrm{~h}$ transfection, the culture was replaced with fresh complete medium.
Western blotting. Cells were lysed with RIPA Lysis Buffer (Sangon Biotech, Co., Ltd.) supplemented with Halt Protease Inhibitor Cocktail (cat. no. 87786, Thermo Fisher Scientific, Inc.). The lysate was centrifuged for $5 \mathrm{~min}$ at $12,000 \mathrm{x} \mathrm{g}$ to remove insoluble substances. Protein concentration was detected by using bicinchoninic acid assay Kit (Sangon Biotech, Co., Ltd.). Proteins (30 $\mu \mathrm{g})$ were separated by $10 \%$ SDS-PAGE and transferred onto polyvinylidene fluoride membranes (EMD Millipore). Membranes were blocked with 5\% skimmed milk for $1 \mathrm{~h}$, incubated with primary antibodies at $4^{\circ} \mathrm{C}$ overnight and with secondary antibodies at for $1 \mathrm{~h}$ at room temperature. The antibodies used were as follows: Anti- Cyto C (1:1,000; cat. no. 11940; Cell Signaling Technology, Inc.); anti-GAPDH (1:1,000; cat. no. 5174; Cell Signaling Technology, Inc.); and anti-cytochrome c oxidase subunit 4I1 (COXIV) antibody (1:1,000; cat. no. ab33985; Abcam). SuperSignal West Femto Maximum Sensitivity Substrate (Thermo Fisher Scientific, Inc.) was used to detect the signal on the membranes, and signal was visualized using a Chemiluminescence Imaging System (Fusion Solo S).

Cell apoptosis assay. To assess the extent of apoptosis, the FITC-Annexin V/Dead Cell Apoptosis kit (Thermo Fisher Scientific, Inc.) with FITC-Annexin V and propidium iodide (PI) double staining was used. A total of $10 \mu \mathrm{l}$ Annexin V and $1 \mu \mathrm{l}$ PI $(100 \mu \mathrm{g} / \mathrm{ml})$ were added to $100 \mu \mathrm{l}$ of cell suspension containing $1 \times 10^{5}$ cells. Following incubation for $30 \mathrm{~min}$ at $37^{\circ} \mathrm{C}$, $400 \mu \mathrm{l}$ binding buffer was added to each tube, and cells were analyzed using a FACSArray flow cytometer (BD Biosciences) Data were analyzed using FlowJo 7.6 software (FlowJo LLC).

Cell Counting Kit-8 (CCK-8) assay. The mock and OECyto C cells were seeded into 96 -well plates at a concentration of 500 cells/well, and cultured for $0,24,48,72$ and 96 h. A solution of $10 \mu \mathrm{l} \mathrm{CCK-8} \mathrm{(Beyotime} \mathrm{Institute} \mathrm{of} \mathrm{Biotechnology)} \mathrm{and}$ $90 \mu \mathrm{l}$ DMEM was added to each well and incubated for $1 \mathrm{~h}$ at $37^{\circ} \mathrm{C}$. Measurements of optical density were collected at $480 \mathrm{~nm}$ using a Multiskan Spectrum reader.

Xenografts. Female, 4-week-old athymic nude mice (Animal Research Center of Nanjing University; $\mathrm{n}=5$ weight, $18 \mathrm{~g}$ ) were housed in a pathogen-free environment $\left(37^{\circ} \mathrm{C}, 5 \% \mathrm{CO}_{2}, 12-\mathrm{h}\right.$ light-dark cycle) and had free access to water and food. The protocol was approved by the Institutional Animal Care and Use Committee of Suqian First Hospital (Suqian, China). A total of $2 \times 10^{6}$ cells overexpressing Cyto $\mathrm{C}$ or mock cells were suspended in $50 \mu \mathrm{l}$ PBS and subcutaneously implanted in the mice. Tumors were detected via bioluminescence imaging using an in vivo Image System Spectrum (PerkinElmer, Inc). The tumor size was measured every 7 days for a month using a Vernier caliper and the volume was calculated as follows: Shortest diameter ${ }^{2}$ $\mathrm{x}$ longest diameter/2. The tumor-bearing mice were sacrificed 4 weeks after implantation, and the tumors were collected and weighed. The xenografts were fixed with $4 \%$ paraformaldehyde for $4 \mathrm{~h}$ at $37^{\circ} \mathrm{C}$ and paraffin embedded. Sections $(4 \mu \mathrm{m})$ from the paraffin block were deparaffinized in xylene and rehydrated in a descending gradient $(100,95,85$ and $75 \%)$ of ethanol. Sections were then boiled in $10 \mathrm{mM}$ citrate buffer, for $15 \mathrm{~min}$ in a microwave oven, and endogenous peroxidase activity was blocked with $0.3 \%$ hydrogen peroxide and $0.1 \%$ saponin 
A

A

$$
\frac{1}{T N} \frac{2}{T N} \frac{3}{T N} \frac{4}{T N} \frac{5}{T N}
$$

Cyto C

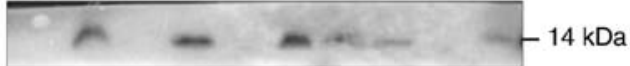

GAPDH

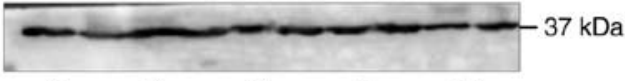

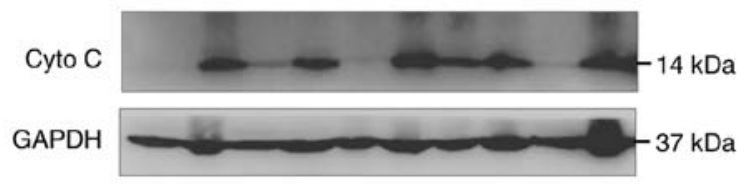

C

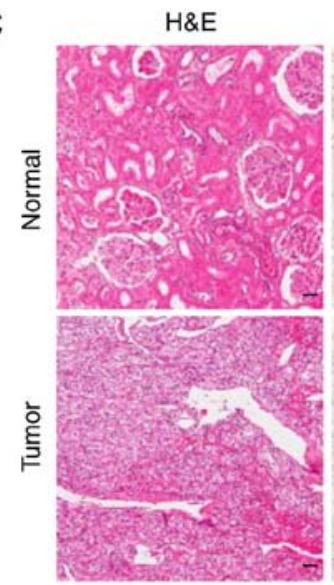

$40 \times$

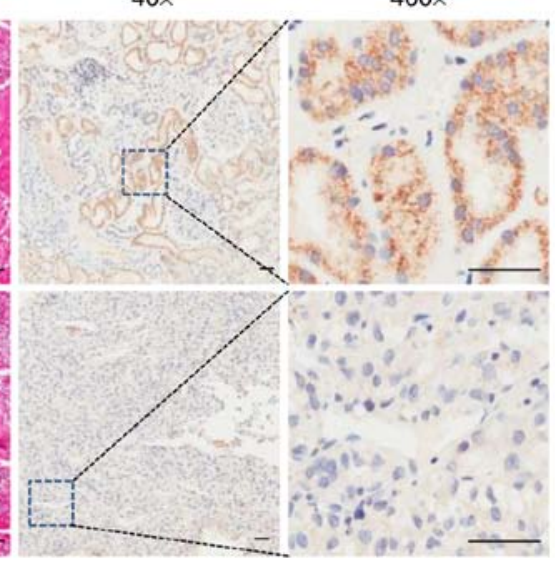

B

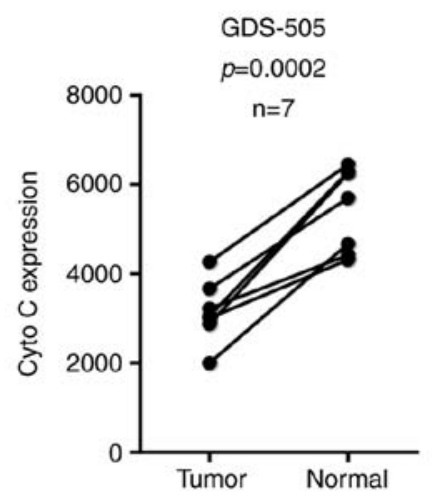

D

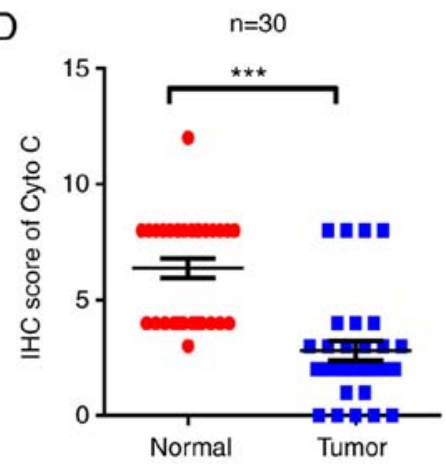

Figure 1. Cyto C expression is decreased in human CCRCC. (A) Cyto C protein expression levels were examined by western blotting in tumor and adjacent normal tissues from 10 patients with CCRCC. (B) mRNA expression levels of Cyto C in seven matched specimens from the Gene Expression Omnibus database GDS-505. (C) Representative images of IHC analysis for Cyto C in normal renal tissues and CCRCC tissues. Scale bars, $30 \mu \mathrm{m}$. (D) IHC scores of tumor and normal tissues from 30 paired CCRCC specimens. ${ }^{* * *} \mathrm{P}<0.001$. Cyto $\mathrm{C}$, cytochrome C; CCRCC, clear renal cell carcinoma; IHC, immunohistochemistry; T, tumor; N, normal; H\&E, hematoxylin and eosin.

dissolved in TBS for $30 \mathrm{~min}$ at $37^{\circ} \mathrm{C}$. Sections were incubated with ki67 antibody (1:100; cat. no. ab15580; Abcam) at $4^{\circ} \mathrm{C}$ overnight, and incubated with avidin-biotin-peroxidase (1:1; cat. no. COD-Nr5007; Dako; Agilent Technologies, Inc) at $37^{\circ} \mathrm{C}$ for $40 \mathrm{~min}$. Signal was visualized with the 3 '-diaminobenzidine visualization kit (Dako; Agilent Technologies, Inc.) and images were captured with an optical microscope (magnification, $\mathrm{x} 40$ ).

Statistical analysis. Statistical analyses were conducted using SPSS software (v19.0; IBM Corp.). The association between Cyto $\mathrm{C}$ expression and the clinicopathological characteristics of patients with CCRCC was assessed by the Pearson $\chi^{2}$ test. The data were presented as the means \pm standard deviation $(\mathrm{n}=3)$. Kaplan-Meier analysis and log-rank test were used to explore the prognostic relevance of Cyto $\mathrm{C}$ in univariate analysis. The statistical software X-tile (version 3.6.1) was used to determine the cutoff in the 150-cohort of CRCC (9). Student's t-test for two groups and one-way ANOVA followed by Least Significant Difference test for multiple groups were applied. $\mathrm{P}<0.05$ was considered to indicate a statistically significant difference.

\section{Results}

Cyto C expression is downregulated in CCRCC tissues. To determine the Cyto $\mathrm{C}$ status in CCRCC, 10 CCRCC and corresponding normal tissues were examined using western blotting. It was revealed that Cyto $\mathrm{C}$ protein expression levels were decreased in CCRCC tissues compared with the corresponding normal tissues (Fig. 1A). In addition, analysis of the previously published GEO database GDS-505 revealed that Cyto C mRNA levels were significantly decreased in CCRCC tissues compared with normal tissues (Fig. 1B). Furthermore, in the 30 paired tissues from the cohort of 150 patients with CCRCC included in the commercial tissue microarray used in the present study, Cyto $\mathrm{C}$ was downregulated in the tumor tissues compared with their corresponding normal tissues (Fig. $1 \mathrm{C}$ and D). In addition, the proportion of Cyto $\mathrm{C}^{\text {high }}$ cells in CCRCC tissues (36.00\%; 54/150) was significantly lower compared with that in the corresponding normal tissues $(96.67 \% ; 29 / 30$; Table I). Overall, these data suggested that low expression levels of Cyto $\mathrm{C}$ may be associated with CCRCC carcinogenesis.

Cyto C confers favorable prognosis in CCRCC. The association between Cyto $\mathrm{C}$ expression and clinicopathological characteristics was investigated in patients with CCRCC. It was revealed that low expression levels of Cyto $\mathrm{C}$ were associated with worse TNM stage ( $\mathrm{P}=0.001$, Table II; and Fig. 2A and B) and tumor depth $(\mathrm{P}=0.008$; Table II). However, there was no significant difference observed in Cyto $\mathrm{C}$ expression levels 
Table I. Cyto $\mathrm{C}$ expression in renal carcinoma and adjacent normal tissues from the tissue microarray analysis.

\begin{tabular}{lccc}
\hline Tissue & Cyto C (low), & Cyto C (high), n & P-value \\
\hline CCRCC cancer & 96 & 54 & \\
Paracancerous & 1 & 29 & $<0.001$ \\
\hline
\end{tabular}

Cyto C, cytochrome C.

among patients with well-, moderately- and poorly-differentiated tumors (Table II). Furthermore, the Kaplan-Meier survival analysis of the 150 CCRCC specimens from the tissue microarray $(\mathrm{P}=0.049$; Fig. $2 \mathrm{C})$ and TCGA database $(\mathrm{P}<0.01$; Fig. 2D) suggested that patients with low Cyto $C$ expression exhibited shorter overall survival rates compared with those with high Cyto $\mathrm{C}$ levels. Univariate and multivariate analyses revealed that decreased Cyto $\mathrm{C}$ expression was an independent prognostic risk factor in CCRCC (hazard ratio, 0.539; 95\% CI, 0.214-1.357; $\mathrm{P}=0.045$; Table III). Therefore, the data of the present study indicated that Cyto $\mathrm{C}$ may be a novel prognostic biomarker for CCRCC.

Cyto $C$ decreases proliferation and increases apoptosis of CCRCC cells in vitro. In order to explore the function of Cyto $\mathrm{C}$ in CCRCC cells, the effects of Cyto $\mathrm{C}$ overexpression and knockdown on the growth and apoptosis of CCRCC cells were investigated. Western blotting confirmed that overexpression and knockdown of Cyto $\mathrm{C}$ protein levels in 786-O cells were achieved (Fig. 3A). As a control, the expression levels of COXIV, a house-keeping gene in mitochondria, were not significantly altered following Cyto C-overexpression or knockdown in 786-O cells (Fig. S2). It was observed that the cell proliferation rate in OECyto $\mathrm{C} 786-\mathrm{O}$ cells was significantly decreased compared with the mock cells (Fig. 3C). By contrast, cell proliferation was significantly increased following Cyto $\mathrm{C}$ knockdown by siRNA (Fig. 3B). Furthermore, the cell apoptosis rate was significantly increased in OECyto C 786-O cells compared with mock cells (Fig. 3D).

Cyto $C$ is crucial for CCRCC tumor growth in vivo. To further investigate the effect of Cyto $\mathrm{C}$ on CCRCC cells in vivo, a subcutaneous xenograft model was established. Bioluminescent imaging in nude mice detected smaller tumors formed by OECyto C CCRCC cells compared with mock cells after 14 days (Fig. 4A). Furthermore, the xenograft tumors formed by OECyto $\mathrm{C}$ cells had a slower growth rate and smaller average volume and weight compared with those formed by mock cells (Fig. 4B-D). In addition, the xenografted tumors derived from the OECyto $C$ cells had decreased proliferation, as indicated by Ki67 staining (Fig. 4E). Overall, these results indicated that overexpression of Cyto $\mathrm{C}$ inhibited the tumorigenicity of CCRCC cells in vivo.

\section{Discussion}

Apoptosis or programmed cell death is vital for the stability of cells, which is associated with several diseases, including
Table II. Association between Cyto $\mathrm{C}$ expression and clinicopathological features of patients with RCC.

\begin{tabular}{|c|c|c|c|}
\hline \multirow[b]{2}{*}{ Feature } & \multicolumn{2}{|c|}{ Cyto $\mathrm{C}$ expression } & \multirow[b]{2}{*}{ P-value } \\
\hline & $\begin{array}{l}\text { Low, n } \\
(n=96)\end{array}$ & $\begin{array}{l}\text { High, } n \\
(n=54)\end{array}$ & \\
\hline Sex & & & 0.845 \\
\hline Male & 69 & 38 & \\
\hline Female & 27 & 16 & \\
\hline Age at diagnosis, years & & & 0.290 \\
\hline$<50$ & 21 & 16 & \\
\hline$\geq 50$ & 75 & 38 & \\
\hline Location & & & 0.859 \\
\hline Left & 43 & 25 & \\
\hline Right & 53 & 29 & \\
\hline T stage & & & 0.008 \\
\hline $\mathrm{T} 1$ & 72 & 50 & \\
\hline T2-T3 & 24 & 4 & \\
\hline $\mathrm{N}$ stage & & & 0.134 \\
\hline N0 & 93 & 54 & \\
\hline $\mathrm{N} 1-2$ & 3 & 0 & \\
\hline TNM & & & 0.001 \\
\hline I & 78 & 44 & \\
\hline II+III & 18 & 10 & \\
\hline Histological grade & & & 0.431 \\
\hline Well & 41 & 21 & \\
\hline Moderate & 44 & 25 & \\
\hline Poor & 11 & 8 & \\
\hline
\end{tabular}

Cyto C, cytochrome C.

a number of different types of cancer, such as CCRCC (10). The mitochondrial signaling pathway, a major apoptosis signaling pathway, is involved in releasing Cyto $\mathrm{C}$ and apoptosis-inducing factors from mitochondria via the $\mathrm{Bcl}-2 / \mathrm{Bax}$ axis (11-13), thereby activating downstream apoptotic executors. Therefore, Cyto $\mathrm{C}$ may be involved in cancer initiation and progression. Although serum Cyto $\mathrm{C}$ has been shown to be a precise indicator of cell death and decreased tumor size following the first cycle of chemotherapy (14), to the best of our knowledge, the relevance and function of Cyto C in CCRCC prior to chemotherapy remains to be elucidated. In the present study, Cyto C expression was demonstrated to be decreased at both the mRNA and protein levels in CCRCC tissues, which suggested that decreased Cyto $\mathrm{C}$ may be involved in CCRCC tumorigenesis.

One of the main features of cancer is to evade apoptosis. Oncogenic events, such as downregulated tumor suppressor genes or upregulated oncogenes that disrupt apoptosis, cause tumor initiation, progression and metastasis (15). In the process of apoptosis, Bcl-2, an anti-apoptotic factor and oncoprotein, prevents Cyto $\mathrm{C}$ release into the cytoplasm from the mitochondrial inner membrane (16). The release 
Table III. Univariate and multivariate analyses for overall survival in clear renal cell carcinoma.

\begin{tabular}{|c|c|c|c|c|}
\hline \multirow[b]{2}{*}{ Factors } & \multicolumn{2}{|c|}{ Univariate } & \multicolumn{2}{|c|}{ Multivariate } \\
\hline & HR $(95 \% \mathrm{CI})$ & P-value & HR (95\% CI) & P-value \\
\hline Sex & $2.594(0.900-7.477)$ & 0.078 & $1.709(0.570-5.217)$ & 0.339 \\
\hline Age & $0.333(0.101-1.031)$ & 0.071 & $0.413(0.124-1.378)$ & 0.150 \\
\hline Location & $1.084(0.513-2.292)$ & 0.832 & $0.896(0.416-1.928)$ & 0.778 \\
\hline Cytochrome $\mathrm{C}$ expression & $0.440(0.178-1.085)$ & 0.049 & $0.539(0.214-1.357)$ & 0.045 \\
\hline Grade & $2.604(1.516-4.472)$ & 0.001 & $1.764(0.951-3.271)$ & 0.072 \\
\hline TNM stage & $3.507(2.313-5.316)$ & $<0.001$ & $3.546(2.336-5.381)$ & $<0.001$ \\
\hline
\end{tabular}

HR, hazard ratio.

A

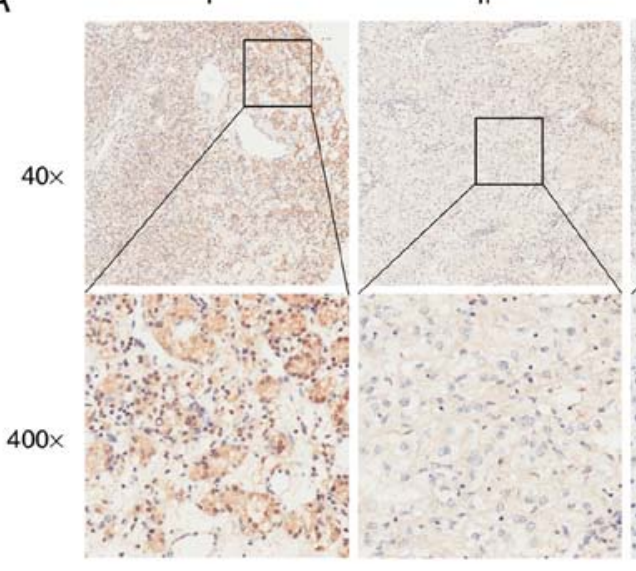

C

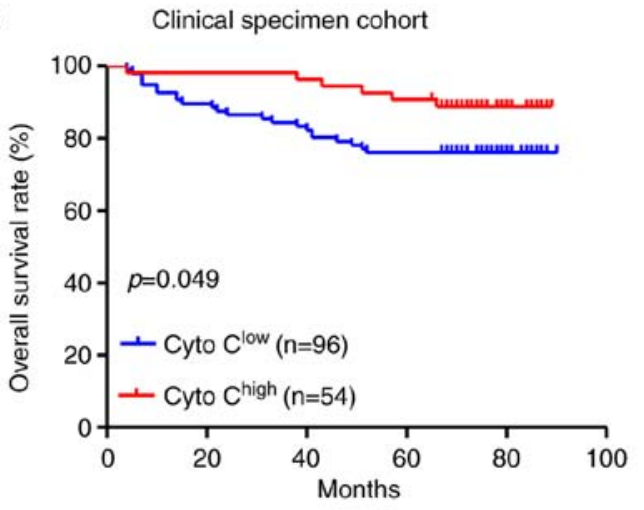

III

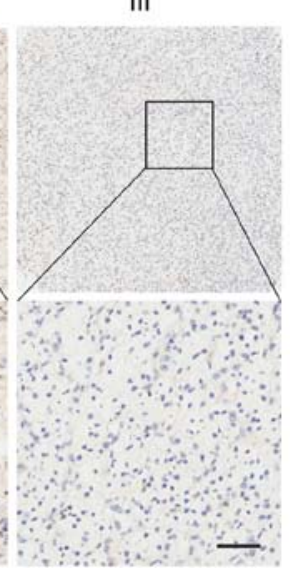

B

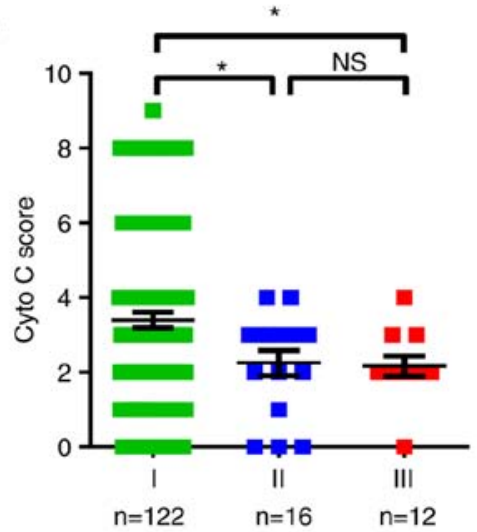

D

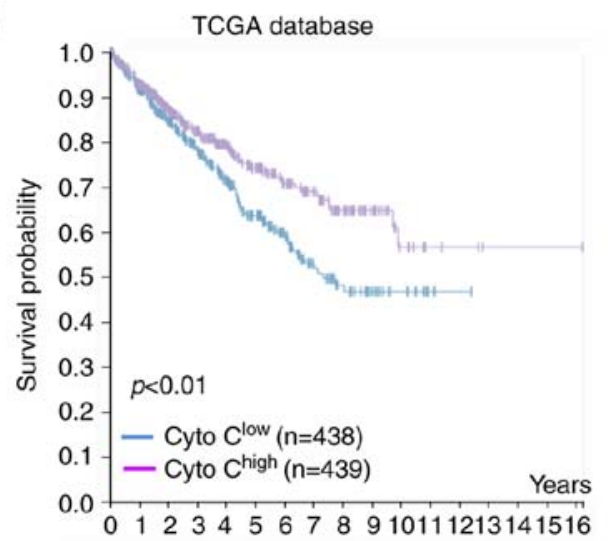

Figure 2. Low expression levels of Cyto C are associated with advanced TNM stage and predict poor prognosis in CCRCC. (A) Representative images of Cyto C staining in CCRCC specimens at different TNM stages. Scale bar, $20 \mu \mathrm{m}$. (B) Immunohistochemistry scores of Cyto C expression in CCRCC tumor tissues of different stages. (C) Kaplan-Meier analysis of overall survival rate in the cohort of 150 patients with CCRCC from the tissue microarray used in the present study. (D) Kaplan-Meier analysis of overall survival rate in a cohort of 877 patients with CCRCC from the TCGA database. "P<0.05. Cyto C, cytochrome C; CCRCC, clear renal cell carcinoma; TCGA, The Cancer Genome Atlas; NS, not significant.

of Cyto $\mathrm{C}$ triggers the formation of an apoptosome and cell death cascade via binding to the apoptosis regulator apoptotic peptidase activating factor 1 (16). The results of the present study revealed that Cyto $\mathrm{C}$ may be involved in tumor growth by altering cell apoptosis. Previous studies have demonstrated that serum Cyto $\mathrm{C}$ levels are lower prior to treatment compared with after chemotherapy and radiotherapy (14). However, another study has demonstrated that serum
Cyto C (17), which is decreased in patients with non-small cell lung carcinoma (NSCLC) prior to treatment compared with healthy individuals, is associated with low TNM stage and a favorable prognosis in NSCLC. The results of the present study demonstrated that low expression levels of Cyto $\mathrm{C}$ were associated with advanced TNM stage and poor prognosis, indicating that Cyto $\mathrm{C}$ was a tumor suppressor gene, and may be a prognostic biomarker in CCRCC. Together with the 

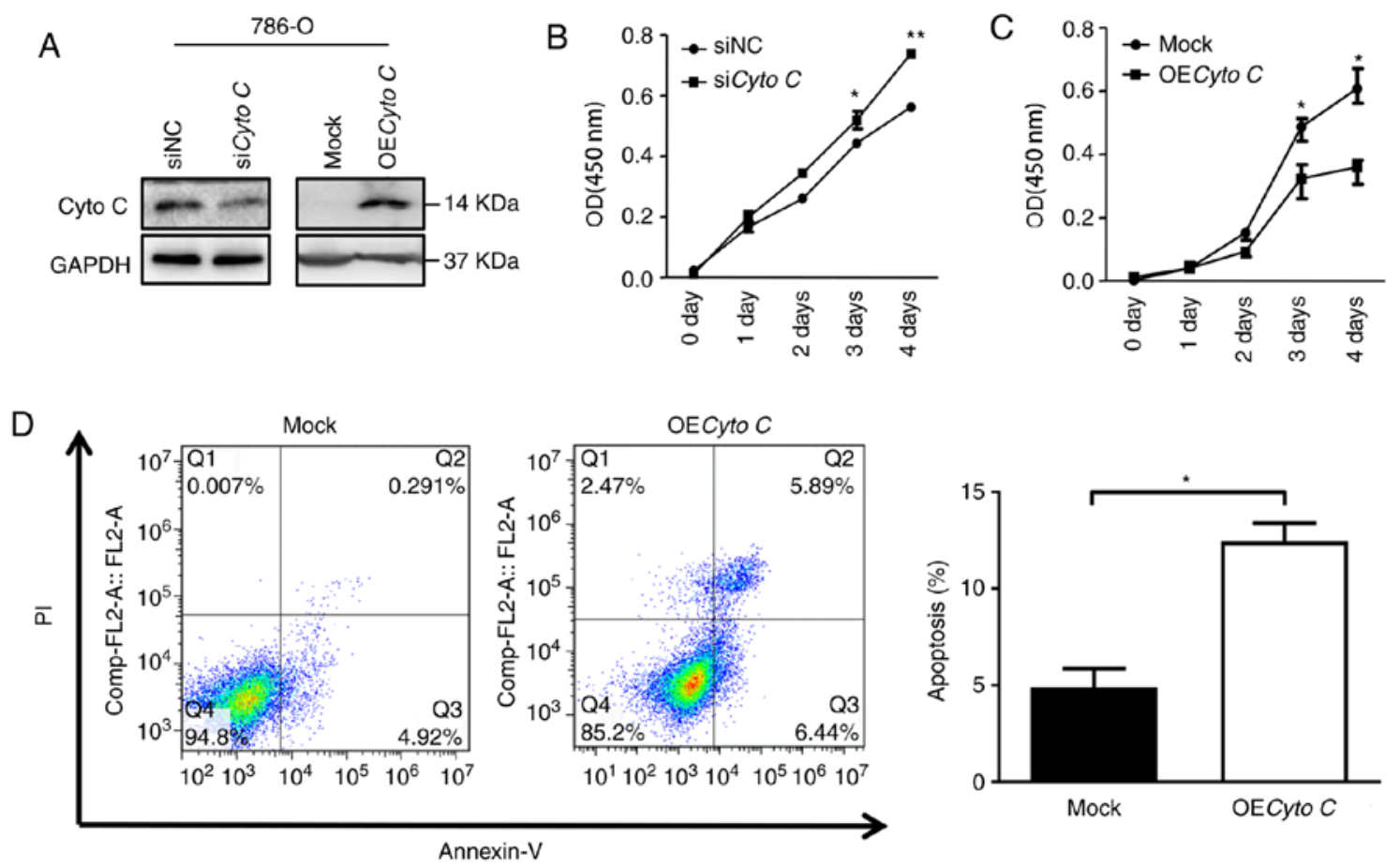

Figure 3. Cyto $\mathrm{C}$ inhibits clear renal cell carcinoma cell proliferation and induces cell apoptosis in vitro. (A) Western blot analysis of Cyto C expression levels in the mock and OECyto C cells, and in the siNC and siCyto C 786-O cells. (B) Cell viability curve of siNC and siCyto C 786-O cells. (C) Cell viability curve of mock and OECyto C 786-O cells. (D) Cell apoptosis was determined via Annexin V-FITC/PI staining and flow cytometry. Data are presented as the mean \pm SD of three independent experiments. " $\mathrm{P}<0.05 ;{ }^{* *} \mathrm{P}<0.01$. Cyto $\mathrm{C}$, cytochrome $\mathrm{C} ; \mathrm{OE}$, overexpression; si, small interfering; $\mathrm{NC}$, negative control; PI, propidium iodide.

A
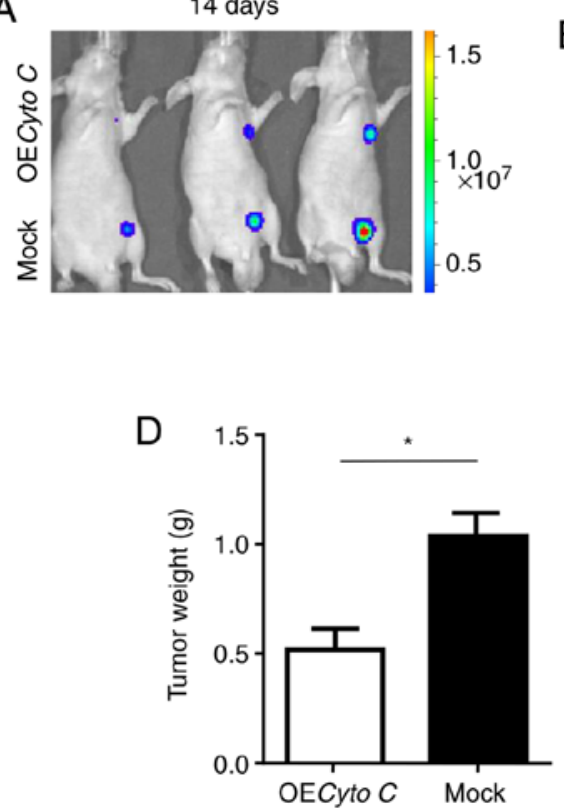

B

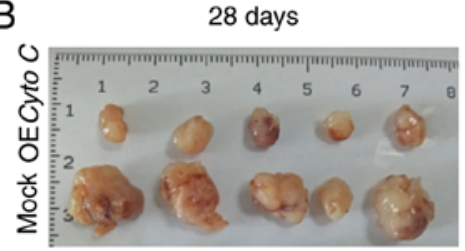

$E$

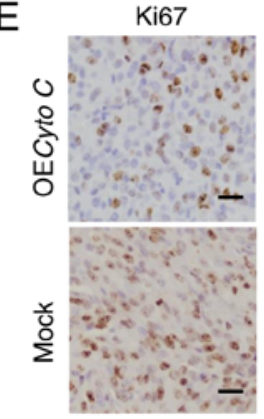

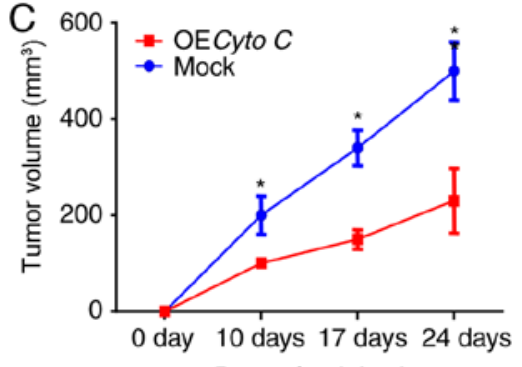

Days after injection

Figure 4. Cyto C suppresses CCRCC cell xenograft growth in vivo. (A) Bioluminescent images of tumors in nude mice implanted with OECyto C and mock CCRCC cells at 14 days. (B) Photographs of the harvested xenograft tumors at 28 days post-implantation. (C) Volume and (D) weight of tumors formed by mock and OECyto C 786-O cells in mice. (E) Representative images of Ki67 staining in sections from the xenograft tumors and quantification of the proliferation index. Scale bar, $20 \mu \mathrm{m}$. Ki67-positive cells (brown) were counted using microscopy and recognized by total number of nuclei in three high-powered fields. The results were presented as the mean number of Ki67-positive cells \pm standard deviation. ${ }^{~} \mathrm{P}<0.05$. Cyto C, cytochrome C; CCRCC, clear renal cell carcinoma; OE, overexpression.

results from previous studies $(14,17)$, this provides improved knowledge of the association between Cyto $\mathrm{C}$ expression and tumor progression.
Unlimited cell proliferation and cell apoptosis are two major characteristics of various types of cancer (18). In the present study, Cyto $\mathrm{C}$ acted as a tumor suppressor gene to 
decrease the proliferation of 786-O in vitro and in xenograft tumors in vivo. Overexpression of Cyto $\mathrm{C}$ in CCRCC cells resulted in decreased growth and increased cancer cell apoptosis in vitro. These data indicated that Cyto $\mathrm{C}$ could serve as a therapeutic target for the treatment of cancer. Furthermore, considering that Cyto $\mathrm{C}$ is a protein with high selectivity and specificity (19), it is an attractive substitute as a cytotoxic drug. In addition, a previous study revealed that $\mathrm{Cyto} C \mathrm{Can}$ be packed with nano-sized smart drug delivery systems to induce apoptosis as a method of cancer treatment (20). The results from the present study provided evidence to further suggest that Cyto C may be a useful and selective therapeutic drug.

In summary, the present study indicated an important function of Cyto $\mathrm{C}$ in controlling the malignant behavior of CCRCC cells, as well as its prognostic value in CCRCC. Therefore, Cyto $\mathrm{C}$ may be a promising therapeutic drug for clinical treatment of CCRCC.

\section{Acknowledgements}

Not applicable.

\section{Funding}

The present study was supported by Suqian Guiding Science and Technology Project (grant no. Z2018174) and Jiangsu Youth Medical Talents Project (grant no. QNRC2016480).

\section{Availability of data and materials}

The datasets used and/or analyzed during the current study are available from the corresponding author on reasonable request.

\section{Authors' contributions}

$\mathrm{ZL}$ and $\mathrm{XZ}$ performed experiments and contributed to the conception of the study. LZ and BP analyzed the data and wrote the manuscript. XZ revised the article. All authors read and approved the final manuscript.

\section{Ethics approval and consent to participate}

The present study was approved by the Ethical Committee of Suqian First Hospital (Suqian Branch Jiangsu Province Hospital). The requirement to obtain patient consent for participation was waived.

\section{Patient consent for publication}

Not applicable.

\section{Competing interests}

The authors declare that they have no competing interests.

\section{References}

1. Moch H, Montironi R, Lopez-Beltran A, Cheng L and Mischo A: Oncotargets in different renal cancer subtypes. Curr Drug Targets 16: 125-135, 2015.
2. Mijuskovic M, Stanojevic I, Milovic N, Cerovic S, Petrovic D, Maksic D, Kovacevic B, Andjelic T, Aleksic P, Terzic B, et al: Tissue and urinary KIM-1 relate to tumor characteristics in patients with clear renal cell carcinoma. Int Urol Nephrol 50: 63-70, 2018.

3. Manickam P, Kaushik A, Karunakaran C and Bhansali S: Recent advances in cytochrome c biosensing technologies. Biosens Bioelectron 87: 654-668, 2017.

4. Renz A, Berdel WE, Kreuter M, Belka C, Schulze-Osthoff K and Los M: Rapid extracellular release of cytochrome $\mathrm{c}$ is specific for apoptosis and marks cell death in vivo. Blood 98: 1542-1548, 2001.

5. Wen Q, Zhang X, Cai J and Yang PH: A novel strategy for real-time and in situ detection of cytochrome c and caspase-9 in Hela cells during apoptosis. Analyst 139: 2499-2506, 2014.

6. Barczyk K, Kreuter M, Pryjma J, Booy EP, Maddika S, Ghavami S, Berdel WE, Roth J and Los M: Serum cytochrome c indicates in vivo apoptosis and can serve as a prognostic marker during cancer therapy. Int J Cancer 116: 167-173, 2005.

7. Osaka A, Hasegawa H, Tsuruda K, Inokuchi N, Yanagihara K, Yamada Y, Aoyama M, Sawada T and Kamihira S: Serum cytochrome $\mathrm{c}$ to indicate the extent of ongoing tumor cell death. Int J Lab Hematol 31: 307-314, 2009.

8. Medeiros LJ, Jones EC, Aizawa S, Aldape HC, Cheville JC, Goldstein NS, Lubensky IA, Ro J, Shanks J, Pacelli A and Jung SH: Grading of renal cell carcinom a: Workgroup No. 2. union internationale contre le cancer and the American Joint Committee on cancer (AJCC). Cancer. Sep 80: 990-991, 1997.

9. Lenburg ME, Liou LS, Gerry NP, Frampton GM, Cohen HT and Christman MF: Previously unidentified changes in renal cell carcinoma gene expression identified by parametric analysis of microarray data. BMC Cancer 3: 31, 2003.

10. Camp RL, Dolled-Filhart $M$ and Rimm DL: X-tile: A new bio-informatics tool for biomarker assessment and outcome-based cut-point optimization. Clin Cancer Res 10: 7252-7259, 2004.

11. Kao SJ, Lee WJ, Chang JH, Chow JM, Chung CL, Hung WY and Chien MH: Suppression of reactive oxygen species-mediated ERK and JNK activation sensitizes dihydromyricetin-induced mitochondrial apoptosis in human non-small cell lung cancer. Environ Toxicol 32: 1426-1438, 2017.

12. Irizarry Rovira AR, Bennet BM, Bolon B, Braendli-Baiocco A, Chandra S, Fleurance R, Garman R, Hutto D, Lane J, Romeike A, et al: Scientific and regulatory policy committee points to consider: Histopathologic evaluation in safety assessment studies for PEGylated pharmaceutical products. Toxicol Pathol 46: 616-635, 2018.

13. Trotta AP and Chipuk JE: Mitochondrial dynamics as regulators of cancer biology. Cell Mol Life Sci 74: 1999-2017, 2017.

14. Kadam CY and Abhang SA: Serum levels of soluble Fas ligand, granzyme B and cytochrome $\mathrm{c}$ during adjuvant chemotherapy of breast cancer. Clin Chim Acta 438: 98-102, 2015.

15. Vladimirov YA, Sarisozen C, Vladimirov GK, Filipczak N, Polimova AM and Torchilin VP: The cytotoxic action of cytochrome C/Cardiolipin nanocomplex (Cyt-CL) on cancer cells in culture. Pharm Res 34: 1264-1275, 2017.

16. Hockenbery D, Nunez G, Milliman C, Schreiber RD and Korsmeyer SJ: Bcl-2 is an inner mitochondrial membrane protein that blocks programmed cell death. Nature 348: 334-336, 1990.

17. Javid J1, Mir R, Julka PK, Ray PC, Saxena A: Extracellular cytochrome $\mathrm{c}$ as a biomarker for monitoring therapeutic efficacy and prognosis of non-small cell lung cancer patients. Tumour Biol 36: 4253-4260,2015.

18. Chen Q, Weng HY, Tang XP, Lin Y, Yuan Y, Li Q, Tang Z, Wu HB, Yang S, Li Y, et al: ARL4C stabilized by AKT/mTOR pathway promotes the invasion of PTEN-deficient primary human glioblastoma. J Pathol 247: 266-278, 2019.

19. Loo JF, Lau PM, Kong SK and Ho HP: An assay using localized surface plasmon resonance and gold nanorods functionalized with aptamers to sense the cytochrome-c released from apoptotic cancer cells for anti-cancer drug effect determination. micromachines (Basel) 8: pii: E338, 2017.

20. Kim ST, Lee YJ, Hwang YS and Lee S: Study on aggregation behavior of Cytochrome C-conjugated silver nanoparticles using asymmetrical flow field-flow fractionation. Talanta 132: 939-944, 2015. 